\title{
CONVERGENCE OF SPECTRAL METHODS FOR NONLINEAR CONSERVATION LAWS*
}

\author{
EITAN TADMOR $\dagger$
}

\begin{abstract}
We discuss the convergence of Fourier methods for scalar nonlinear conservation laws that exhibit spontaneous shock discontinuities. Numerical tests indicate that the convergence may (and in fact in some cases we prove it must) fail, with or without post-processing of the numerical solution. Instead, we introduce here a new kind of spectrally accurate vanishing viscosity to augment the Fourier approximation of such nonlinear conservation laws. Using compensated compactness arguments augmented by the assumption of $L^{\infty}$-stability, we show for the inviscid Burgers' model equation that this spectral viscosity method prevents oscillations, and convergence to the unique entropy solution follows.
\end{abstract}

Key words. Burgers' equation, entropy solution, the spectral viscosity method, compensated compactness, convergence

AMS(MOS) subject classifications. 35L65, 65M10, 65M15

1. Introduction. In this paper we study the convergence of spectral methods for nonlinear conservation laws. Specifically, we consider what is accepted by now as the universal model problem for such scalar laws, namely, the inviscid Burgers' equation

$$
\frac{\partial}{\partial t} u(x, t)+\frac{\partial}{\partial x}\left(\frac{u^{2}(x, t)}{2}\right)=0
$$

subject to given initial data $u(x, t=0)$. Among the basic features of solutions to this problem [6], we recall the following: that they may develop spontaneous jump discontinuities (shock waves), and hence the class of weak solutions must be admitted; that within this class, there are many possible solutions; and that in order to single out the unique "physically relevant" solution among them, (1.1) is augmented with an additional entropy condition that requires

$$
\frac{\partial}{\partial t}\left(\frac{u^{2}(x, t)}{2}\right)+\frac{\partial}{\partial x}\left(\frac{u^{3}(x, t)}{3}\right) \leqq 0 .
$$

The existence of physically relevant shock waves in the solution is reflected by the strict (distributional) inequality in (1.2).

We want to solve the $2 \pi$-periodic problem (1.1), (1.2) by the spectral-Fourier method. To this end, we approximate the spectral-Fourier projection of $u(x, t)$,

$$
P_{N} u(x, t)=\sum_{k=-N}^{N} \hat{u}(k, t) e^{\imath k x}, \quad \hat{u}(k, t)=\frac{1}{2 \pi} \int_{-\pi}^{\pi} u(x, t) e^{-\imath k x} d x,
$$

by an $N$-trigonometric polynomial, $u_{N}(x, t)$,

$$
u_{N}(x, t)=\sum_{k=-N}^{N} \hat{u}_{k}(t) e^{i k x}
$$

* Received by the editors September 21, 1987; accepted for publication (in revised form) January 8, 1988. This research was supported in part by National Aeronautics and Space Administration contract NAS1-18107 while the author was in residence at ICASE. Additional support was provided by U.S.-Israel Binational Science Foundation grant 85-00346, National Science Foundation grant DMS85-03294, and Army Research Office grant DAAG-85-K-0190 while the author was in residence at the University of California, Los Angeles, California 90024.

† School of Mathematical Sciences, Tel-Aviv University, Ramat-Aviv, Tel-Aviv, Israel 69 978, and Institute for Computer Applications in Science and Engineering, NASA Langley Research Center, Hampton, Virginia 23665. The author is a Bat-Sheva Foundation Fellow. 
Starting with

$$
u_{N}(x, 0)=P_{N} u(x, 0),
$$

the classical Fourier method [3] lets $u_{N}(x, t)$ evolve at a later time according to the approximate model

$$
\frac{\partial}{\partial t} u_{N}(x, t)+\frac{\partial}{\partial x}\left(\frac{1}{2} P_{N} u_{N}^{2}(x, t)\right)=0 .
$$

Noting that $P_{N}$ commutes with differentiation, we can rewrite (1.6) in the equivalent form:

$$
\frac{\partial}{\partial t} u_{N}(x, t)+\frac{\partial}{\partial x}\left(\frac{1}{2} u_{N}^{2}(x, t)\right)=\left(I-P_{N}\right) \frac{\partial}{\partial x}\left(\frac{1}{2} u_{N}^{2}(x, t)\right) .
$$

Let us multiply (1.7) by $u_{N}(x, t)$ : since $u_{N}(x, t)$ is orthogonal to the right-hand side of (1.7), we find after integration that

$$
\begin{aligned}
\frac{1}{2} \frac{d}{d t} \int_{-\pi}^{\pi} u_{N}^{2}((x, t) d x & =-\int_{-\pi}^{\pi} u_{N}(x, t) \frac{\partial}{\partial x}\left(\frac{1}{2} u_{N}^{2}(x, t)\right) d x \\
& =-\left.\frac{u_{N}^{3}(x, t)}{3}\right|_{x=-\pi} ^{x=\pi}=0 .
\end{aligned}
$$

Thus $\int u_{N}^{2}(x, t) d x$ is conserved in time

$$
\int_{-\pi}^{\pi} u_{N}^{2}(x, t) d x=\int_{-\pi}^{\pi} u_{N}^{2}(x, 0) d x \leqq \int_{-\pi}^{\pi} u^{2}(x, 0) d x,
$$

and this yields the existence of a weak limit $\bar{u}(x, t)=w \lim _{N \rightarrow \infty} u_{N}(x, t)$. Does $\bar{u}(x, t)$ solve our problem? Unfortunately the answer is no. For otherwise, if $\bar{u}(x, t)$ is a weak solution of (1.1), then $P_{N} u_{N}^{2}(x, t)$, and hence $u_{N}^{2}(x, t)$ should tend weakly to $\bar{u}^{2}(x, t)$, and consequently, $\bar{u}(x, t)$ should be the strong limit of $u_{N}(x, t)$; but then (1.9) implies that $\int_{-\pi}^{\pi} \bar{u}^{2}(x, t) d x$ is also conserved in time, and by (1.2) this contradicts the appearance of physically relevant shock waves in our solution.

In practical applications, spectral methods are often augmented with smoothing procedures in order to give a helping hand toward their spectral convergence. Indeed, convergence for smoothed versions of spectral (and in particular pseudospectral) methods, was established in the linear case, e.g., [5], [7], [13]. However, arguments similar to the above show that with nonlinear problems, convergence of the Fourier method fails despite the additional smoothing of its solution. We leave the details for the Appendix. Instead, we propose here a different way to enforce the convergence of the spectral-Fourier method without sacrificing spectral accuracy. This is accomplished by introducing, in $\S 2$, a new type of spectral vanishing viscosity. In $\S 3$ we use compensated compactness arguments augmented with the assumption of $L^{\infty}$-stability in order to prove the convergence of the proposed method to a weak solution of (1.1), and in $\S 4$ we show that this weak solution respects the entropy condition (1.2). This proves that if the spectral viscosity solutions are uniformly bounded, then they will converge to the unique entropy solution of Burgers' equation. In $\S 5$ we extend our discussion to systems of conservation laws, and we show how the spectral vanishing viscosity can be used to enforce the correct entropy dissipation in such a case. Finally, numerical experiments with the proposed method of spectral regularization are presented in $\S 6$. 
2. The spectral vanishing viscosity. It is well known [6] that the unique entropy solution of (1.1), (1.2) is the one identified with the small viscosity limit of the regularized problem

$$
\frac{\partial}{\partial t} u_{\varepsilon}(x, t)+\frac{\partial}{\partial x}\left(\frac{1}{2} u_{\varepsilon}^{2}(x, t)\right)=\varepsilon \frac{\partial}{\partial x}\left[Q \frac{\partial}{\partial x} u_{\varepsilon}(x, t)\right], \quad \varepsilon \downarrow 0 .
$$

With the vanishing viscosity method [9], we replace the exact derivatives in (2.1) by their discrete counterpart, the viscosity coefficient $Q$ is chosen as (a nonlinear) positive grid dependent quantity, and the role of $\varepsilon$ is played by some fixed power of the vanishing grid size, $\varepsilon \sim(\Delta x)^{s}$, in order to yield an $s$-order accurate approximation of (1.1). Yet in order to respect spectral accuracy, a more delicate viscous regularization is required. To this end we consider viscosity coefficients of the form $Q=I-P_{m}$. The resulting viscosity terms are of spectrally small order of magnitude in the sense that for any $s>0$ we have

$$
\left\|\varepsilon \frac{\partial}{\partial x}\left[\left(I-P_{m}\right) \frac{\partial}{\partial x} u(x, t)\right]\right\|_{L^{2}(x)} \leqq \varepsilon\|u\|_{H^{s+2}(x)} \cdot m^{-s}
$$

in fact these terms are exponentially small in the analytic case [10]. Together with this kind of spectral vanishing viscosity, the spectrally accurate Fourier approximation of (1.1) amounts to

$$
\frac{\partial}{\partial t} u_{N}(x, t)+\frac{\partial}{\partial x}\left(\frac{1}{2} P_{N} u_{N}^{2}(x, t)\right)=\varepsilon \frac{\partial}{\partial x}\left[\left(I-P_{m}\right) \frac{\partial}{\partial x} u_{N}(x, t)\right],
$$

and we raise the question of its convergence as $N$ tends to infinity. Here $\varepsilon \equiv \varepsilon(N) \downarrow 0$ and $m \equiv m(N)<N$ are free parameters that are yet to be determined, subject to the spectral accuracy restriction $m(N) \uparrow \infty$. In the next two sections we find such admissible parameters that provide a positive answer to the convergence question.

3. Spectral convergence to a weak solution. We consider the approximate Fourier method (2.2), which we rewrite as

$$
\frac{\partial}{\partial t} u_{N}+\frac{\partial}{\partial x}\left(\frac{1}{2} u_{N}^{2}\right)=\frac{\partial}{\partial x}\left[\left(I-P_{N}\right) \frac{1}{2} u_{N}^{2}\right]+\varepsilon \frac{\partial}{\partial x}\left[\left(I-P_{m}\right) \frac{\partial}{\partial x} u_{N}\right] \equiv I+I I .
$$

In order to prove convergence of this method, we need a couple of a priori estimates on its solution. To this end, we multiply (3.1) by $u_{N}$,

$$
\begin{aligned}
\frac{\partial}{\partial t}\left(\frac{1}{2} u_{n}^{2}\right)+\frac{\partial}{\partial x}\left(\frac{1}{3} u_{N}^{3}\right) & =u_{N} \frac{\partial}{\partial x}\left[\left(I-P_{N}\right) \frac{1}{2} u_{N}^{2}\right]+\varepsilon u_{N} \frac{\partial}{\partial x}\left[\left(I-P_{m}\right) \frac{\partial}{\partial x} u_{N}\right] \\
& \equiv I I I+I V
\end{aligned}
$$

and integrate over the $2 \pi$ period: the integrals of the second and third terms vanish by periodicity and orthogonality, and we are left with

$$
\frac{1}{2} \frac{d}{d t}\left\|u_{N}(\cdot, t)\right\|_{L^{2}(x)}^{2}+\varepsilon\left\|\left(I-P_{m}\right) \frac{\partial}{\partial x} u_{N}(\cdot, t)\right\|_{L^{2}(x)}^{2}=0 .
$$

This gives us the a priori bound on the amplitudes of the solution we had before in (1.9), and even a little more. More precisely, temporal integration of (3.3) yields

$$
\begin{aligned}
& \frac{1}{2}\left\|u_{N}(\cdot, t)\right\|_{L^{2}(x)}^{2}+\varepsilon \int_{\tau=0}^{t}\left\|\left(I-P_{m}\right) \frac{\partial}{\partial x} u_{N}(\cdot, \tau)\right\|_{L^{2}(x)}^{2} d \tau \\
& =\frac{1}{2}\left\|u_{N}(\cdot, t=0)\right\|_{L^{2}(x)}^{2},
\end{aligned}
$$


and hence for $u_{N}(x, t) \equiv \sum_{|k| \leqq N} \hat{u}_{k}(t) e^{i k \cdot x}$ we have

$$
\left\|u_{N}(\cdot, t)\right\|_{L^{2}(x)}^{2}=\sum_{|k| \leqq N}\left|\hat{u}_{k}(t)\right|^{2} \leqq \text { Const }_{1}, \quad \text { Const }_{1}=\|u(\cdot, t=0)\|_{L^{2}(x)}^{2}
$$

equality (3.4) also gives us the second a priori estimate

$$
\varepsilon\left\|\left(I-P_{m}\right) \frac{\partial u_{N}}{\partial x}\right\|_{L_{\text {loc }}^{2}(x, t)}^{2} \equiv \varepsilon \int_{t} \sum_{m<|k| \leqq N} k^{2}\left|\hat{u}_{k}(t)\right|^{2} d t \leqq \text { Const }_{2} .
$$

Equipped with these estimates we may now turn to the convergence proof of the Fourier method (2.2). We will establish spectral convergence for an admissible set of parameters $\varepsilon(N) \downarrow 0, m(N) \uparrow \infty$, using Tartar's div-curl lemma [14]. In order to apply the latter in our case, we have to verify that the four expressions appearing on the right-hand sides of (3.1) and (3.2) are "nice" ones, namely, that these expressions are the sum of terms, each of which lies either in a compact subset of $H_{\mathrm{loc}}^{-1}(x, t)$, or, by Murat's lemma, in a bounded set of $L_{\mathrm{loc}}^{1}(x, t)$. In the following lemmas we collect the necessary estimates in this direction.

We begin with the first term on the right of (3.1). Here, the following estimate, the proof of which is postponed to the end of this section, is essential.

Lemma 3.1. There exists a constant Const $\mathrm{1}_{12}$ (depending on Const ${ }_{1}$ and Const $_{2}$ ) such that for $m<\frac{1}{2} N$ we have

$$
\begin{aligned}
\left\|I \equiv \frac{\partial}{\partial x}\left[\left(I-P_{N}\right) \frac{1}{2} u_{N}^{2}\right]\right\|_{H_{\mathrm{loc}}^{-1}(x, t)} & \leqq\left\|\left(I-P_{N}\right) \frac{1}{2} u_{N}^{2}\right\|_{L_{\mathrm{loc}}^{2}(x, t)} \\
& \leqq \operatorname{Const}_{12} \cdot(\varepsilon N)^{-1 / 2} .
\end{aligned}
$$

Next, we use the a priori estimate (3.6) to conclude that, as $\varepsilon$ tends to zero, the second term on the right of (3.1) belongs to a compact subset of $H_{\mathrm{loc}}^{-1}(x, t)$.

LEMMA 3.2. The following estimate holds:

$$
\begin{aligned}
\left\|I I \equiv \varepsilon \frac{\partial}{\partial x}\left[\left(I-P_{m}\right) \frac{\partial u_{N}}{\partial x}\right]\right\|_{H_{\mathrm{loc}}^{-1}(x, t)} & \leqq \varepsilon\left\|\frac{\partial}{\partial x}\left(I-P_{m}\right) \frac{\partial u_{N}}{\partial x}\right\|_{L_{\mathrm{loc}}^{2}(x, t)} \\
& \leqq\left(\varepsilon \cdot \text { Const }_{2}\right)^{1 / 2} \rightarrow 0, \quad \varepsilon \downarrow 0 .
\end{aligned}
$$

To treat the expressions on the right of (3.2), we must first do some preparation.

Lemma 3.3. There exist constants Const ${ }_{3}$ and Const $_{23}$ (depending on Const ${ }_{2}$ and Const $_{3}$ ) such that

$$
\begin{aligned}
& \left\|P_{m} \frac{\partial^{s} u_{N}}{\partial x^{s}}\right\|_{L_{\mathrm{loc}}^{2}(x, t)} \leqq \mathrm{Const}_{3} \cdot m^{s}, \\
& \left\|\frac{\partial u_{N}}{\partial x}\right\|_{L_{\mathrm{loc}}^{2}(x, t)} \leqq \operatorname{Const}_{23} \cdot\left(\varepsilon^{-1 / 2}+m\right) .
\end{aligned}
$$

Proof. The inequality (3.9) follows immediately in view of $\left.\| P_{m} \partial^{s} u_{N} / \partial x^{s}\right) \|_{L_{\text {loc }}^{2}(x, t)} \leqq$ $m^{s} \cdot\left\|u_{N}\right\|_{L_{\text {loc }}^{2}(x, t)}$ and the a priori estimate (3.5). To prove (3.10) we invoke the identity

$$
\left\|\frac{\partial u_{N}}{\partial x}\right\|_{L_{\text {loc }}^{2}(x, t)}^{2} \equiv\left\|\left(I-P_{m}\right) \frac{\partial u_{N}}{\partial x}\right\|_{L_{\text {loc }}^{2}(x, t)}^{2}+\left\|P_{m} \frac{\partial u_{N}}{\partial x}\right\|_{L_{\text {loc }}^{2}(x, t)}^{2},
$$

and use the a priori estimate (3.6) to upper bound the first norm, and (3.9) for the second one. 
In the next two lemmas we turn to deal with the right-hand side of (3.2). For its first member, which we express as

$$
\begin{aligned}
I I I & \equiv u_{N} \frac{\partial}{\partial x}\left[\left(I-P_{N}\right) \frac{1}{2} u_{N}^{2}\right]=\frac{\partial}{\partial x}\left[u_{N}\left(I-P_{N}\right) \frac{1}{2} u_{N}^{2}\right]-\frac{\partial u_{N}}{\partial x}\left(I-P_{N}\right) \frac{1}{2} u_{N}^{2} \\
& \equiv I I I_{1}+I I I_{2},
\end{aligned}
$$

we have the following lemma.

Lemma 3.4. There exists a constant Const ${ }_{123}$ (depending on Const ${ }_{12}$ and Const $_{23}$ ) such that the following estimates hold:

$$
\begin{aligned}
& \left\|I I I_{1}=\frac{\partial}{\partial x}\left[u_{N}\left(I-P_{N}\right) \frac{1}{2} u_{N}^{2}\right]\right\|_{H_{\mathrm{loc}}^{-1}(x, t)} \leqq \text { Const }_{123} \cdot\left\|u_{N}\right\|_{L^{\infty}(x, t)} \cdot(\varepsilon N)^{-1 / 2}, \\
& \left\|I I I_{2} \equiv-\frac{\partial u_{N}}{\partial x}\left(I-P_{N}\right) \frac{1}{2} u_{N}^{2}\right\|_{L_{\mathrm{loc}}^{1}(x, t)} \leqq \text { Const }_{123} \cdot\left(\varepsilon^{-1 / 2}+m\right) \cdot(\varepsilon N)^{-1 / 2} .
\end{aligned}
$$

Proof. The first estimate, (3.12), follows from Lemma 3.1:

$$
\begin{aligned}
\left\|I I I_{1}\right\|_{H_{\mathrm{loc}}^{-1}(x, t)} & \leqq\left\|u_{N}\left(I-P_{N}\right) \frac{1}{2} u_{N}^{2}\right\|_{L_{\mathrm{loc}}^{2}(x, t)} \\
& \leqq\left\|u_{N}\right\|_{L^{\infty}(x, t)} \cdot\left\|\left(I-P_{N}\right) \frac{1}{2} u_{N}^{2}\right\|_{L_{\mathrm{loc}}^{2}(x, t)} \\
& \leqq \text { Const }_{12} \cdot\left\|u_{N}\right\|_{L^{\infty}(x, t)}(\varepsilon N)^{-1 / 2}
\end{aligned}
$$

together with Lemma 3.3 we also have

$$
\left\|I I I_{2}\right\|_{L_{\mathrm{loc}}^{1}(x, t)} \leqq\left\|\frac{\partial u_{N}}{\partial x}\right\|_{L_{\mathrm{loc}}^{2}(x, t)} \cdot\left\|\left(I-P_{N}\right) \frac{1}{2} u_{N}^{2}\right\|_{L_{\mathrm{loc}}^{2}(x, t)},
$$

and the second estimate, (3.13), follows with $C_{123}=$ Const $_{12} \cdot$ Const $_{23}$.

Finally, the second member on the right of (3.2),

$$
\begin{aligned}
I V & \equiv \varepsilon u_{N}\left(I-P_{m}\right) \frac{\partial^{2} u_{N}}{\partial x^{2}}=\varepsilon \frac{\partial}{\partial x}\left[u_{N}\left(I-P_{m}\right) \frac{\partial u_{N}}{\partial x}\right]-\varepsilon \frac{\partial u_{N}}{\partial x}\left(I-P_{m}\right) \frac{\partial u_{N}}{\partial x} \\
& \equiv I V_{1}+I V_{2},
\end{aligned}
$$

is estimated as follows.

Lemma 3.5. There exists a constant Const $_{32}$ (depending on Const $_{2}$ and Const $_{3}$ ) such that the following estimates hold:

$$
\begin{gathered}
\left\|I V_{1} \equiv \varepsilon \frac{\partial}{\partial x}\left[u_{N}\left(I-P_{m}\right) \frac{\partial u_{N}}{\partial x}\right]\right\|_{H_{\mathrm{loc}}^{-1}(x, t)} \leqq \mathrm{Const}_{32} \cdot\left\|u_{N}\right\|_{L^{\infty}(x, t)} \cdot \varepsilon^{1 / 2}, \\
\left\|I V_{2} \equiv-\varepsilon \frac{\partial u_{N}}{\partial x}\left(I-P_{m}\right) \frac{\partial u_{N}}{\partial x}\right\|_{L_{\mathrm{loc}}^{1}(x, t)} \leqq \mathrm{Const}_{32}\left(1+\varepsilon^{1 / 2} m\right) .
\end{gathered}
$$

Proof. The first inequality, (3.16), follows from the a priori estimate (3.6):

$$
\left\|I V_{1}\right\|_{H_{\mathrm{loc}}^{-1}(x, t)} \leqq \varepsilon\left\|u_{N}\right\|_{L^{\infty}(x, t)} \cdot\left\|\left(I-P_{m}\right) \frac{\partial u_{N}}{\partial x}\right\|_{L_{\mathrm{loc}}^{2}(x, t)} \leqq\left\|u_{N}\right\|_{L^{\infty}(x, t)} \cdot\left(\text { Const }_{2} \cdot \varepsilon\right)^{1 / 2} .
$$

To prove the second inequality we upper bound

$$
\begin{aligned}
\left\|I V_{2}\right\|_{L_{\mathrm{loc}}^{1}(x, t)} \leqq & \varepsilon\left\|\left(I-P_{m}\right) \frac{\partial u_{N}}{\partial x}\right\|_{L_{\mathrm{loc}}^{2}(x, t)}^{2} \\
& +\sqrt{\varepsilon}\left\|P_{m} \frac{\partial u_{N}}{\partial x}\right\|_{L_{\mathrm{loc}}^{2}(x, t)} \cdot \sqrt{\varepsilon}\left\|\left(I-P_{m}\right) \frac{\partial u_{N}}{\partial x}\right\|_{L_{\mathrm{loc}}^{2}(x, t)},
\end{aligned}
$$


and use the a priori estimates (3.6) and (3.9) to obtain (3.16) with Const $_{32}=$ Const $_{2} \cdot\left(\right.$ Const $\left._{3}+1\right)$.

We are now ready to find the admissible parameters, $\varepsilon=\varepsilon(N), m=m(N)$, which meet the assumptions of the div-curl lemma. By (3.16), the term $I V_{2}$ is bounded in $L_{\mathrm{loc}}^{1}(x, t)$ if $\varepsilon^{1 / 2} m \leqq$ Const.; choosing $\varepsilon \sim$ Const./ $m^{2}$, then by (3.13) the term $I I I_{2}$ is bounded in $L_{\mathrm{loc}}^{1}(x, t)$ provided $m^{2} N^{-1 / 2} \leqq$ Const.; choosing $m \leqq \min$ (Const. $N^{1 / 4}, \frac{1}{2} N$ ) we conclude by Lemma 3.1 and Lemma 3.2, that the terms $I$ and $I I$ belong to a compact subset of $H_{\mathrm{loc}}^{-1}(x, t)$. Moreover, if $u_{N}$ is uniformly bounded,

$$
\left\|u_{N}\right\|_{L^{\infty}(x, t)} \text { Const. }
$$

then by Lemmas 3.4 and 3.5 we have with this choice of parameters that the terms $I I I_{1}$ and $I V_{1}$ are also in a compact subset of $H_{\mathrm{loc}}^{-1}(x, t)$. This completes our study of the expressions on the right of (3.1), (3.2) and the div-curl lemma applies in our case. We summarize by stating Theorem 3.6.

THEOREM 3.6. Consider the spectral approximation (2.2) with parameters $(\varepsilon, m)$ that satisfy

$$
\varepsilon=\varepsilon(N) \sim \text { Const. } N^{-2 \beta}, \quad m=m(N) \sim \text { Const. } N^{\beta}, \quad 0<\beta \leqq \frac{1}{4},
$$

Assume that its solution, $u_{N}(x, t)$, remains uniformly bounded, (3.17). Then $u_{N}(x, t)$ converges boundedly almost everywhere to a weak solution of the conservation law (1.1).

Proof. Let $u^{(j)}(x, t)$ denote the weak limit of $u_{N}^{j}(x, t)$,

$$
\underset{N \rightarrow \infty}{w} \lim _{N} u_{N}^{j}(x, t)=\overline{u^{(j)}}(x, t) .
$$

Applying the div-curl lemma to (3.1), (3.2) we have the relation

$$
\overline{u^{(4)}}=4 \overline{u^{(1)}} \cdot \overline{u^{(3)}}-3\left(\overline{u^{(2)}}\right)^{2},
$$

which implies strong convergence. To establish this implication, we follow the argument of Tartar [15] (see also [14]), who suggests considering the weak limit of $\left(u_{N}-\overline{u_{N}^{(1)}}\right)^{4}$,

$$
\left.w_{N \rightarrow \infty} \lim _{N}\left(u_{N}-\overline{u^{(4)}}\right)^{4}=\overline{u^{(4)}}-4 \overline{u^{(3)} u^{(1)}}+6 \overline{u^{(2)}} \overline{\left(u^{(1)}\right.}\right)^{2}-4\left(\overline{u^{(1)}}\right)^{4}+\left(\overline{u^{(1)}}\right)^{4}
$$

using (3.19) and rearranging, we find

$$
\underset{N \rightarrow \infty}{w} \lim _{N}\left(u_{N}-\overline{u^{(1)}}\right)^{4}=-3\left[\overline{u^{(2)}}-\left(\overline{u^{(1)}}\right)^{2}\right]^{2} \leqq 0,
$$

and hence $\overline{u^{(2)}}=\left(\overline{u^{(1)}}\right)^{2}$. Consequently, $u_{N}(x, t)$ converges strongly to $\bar{u}(x, t) \equiv \overline{u^{(1)}}(x, t)$ in $L_{\mathrm{loc}}^{p}(x, t)$, and by (3.1), (3.7), and (3.8), $\bar{u}(x, t)$ is a weak solution of (1.1).

We do not claim that our parametrization (3.18) is optimal. In particular, the restrictive choice of $m(N)$ could be improved as indicated by the numerical tests described in $\S 6$. On the other hand, we note that the $\varepsilon$ parametrization (3.18) such that $\varepsilon m^{2} \sim$ Const. yields, in view of inequality (3.10),

$$
\sqrt{\varepsilon}\left\|\frac{\partial u_{N}}{\partial x}\right\|_{L_{\text {loc }}^{2}(x, t)} \leqq \text { Const. }
$$

which is in complete agreement with the behavior of the viscous regularization model (2.1), where

$$
\sqrt{\varepsilon}\left\|\frac{\partial u_{\varepsilon}}{\partial x}\right\|_{L_{\mathrm{loc}}^{2}(x, t)} \leqq \text { Const. }
$$


Our choice of parameters in (3.18) depends heavily on the essential estimate (3.7), and we conclude this section with its proof.

Proof of Lemma 3.1. We should upper bound the norm of

$$
\begin{aligned}
\left\|\left(I-P_{N}\right) \frac{1}{2} u_{N}^{2}\right\|_{L_{\mathrm{loc}}^{2}(x, t)}^{2}= & \frac{1}{2} \int_{t} \sum_{N<p \leqq 2 N}\left|\sum_{k=p-N}^{N} \hat{u}_{k}(t) \overline{\hat{u}}_{p-k}(t)\right|^{2} \\
& +\frac{1}{2} \int_{t-2 N \leqq p<-N}\left|\sum_{k=-N} \hat{u}_{k}(t) \overline{\hat{u}}_{p-k}(t)\right|^{2} .
\end{aligned}
$$

The first integral on the right does not exceed

$$
\begin{aligned}
\frac{1}{2} \int_{t} \sum_{N<p \leqq 2 N}\left[\left(\sum_{k=p-N}^{p / 2}+\right.\right. & \left.\left.\sum_{k=p / 2}^{N}\right)\left|\hat{u}_{k}(t)\right| \cdot\left|\hat{u}_{p-k}(t)\right|\right]^{2} \\
& =2 \int_{t} \sum_{N<p \leqq 2 N}\left[\sum_{k=p / 2}^{N}\left|\hat{u}_{k}(t)\right| \cdot\left|\hat{u}_{p-k}(t)\right|\right]^{2},
\end{aligned}
$$

and using Cauchy-Schwartz inequality this is less than

$$
2 \int_{t} \sum_{N<p \leqq 2 N}\left(\sum_{k=p / 2}^{N} k^{2}\left|\hat{u}_{k}(t)\right|^{2} \cdot \sum_{k=p / 2}^{N} \frac{1}{k^{2}}\left|\hat{u}_{p-k}(t)\right|^{2}\right) .
$$

According to our assumption $m<\frac{1}{2} N$. Hence for $p>N$ we have $p / 2>m$, and by the a priori estimates (3.5), (3.6), the last expression is bounded from above by

$$
\begin{aligned}
2 \cdot \max _{t}\left(\sum_{N<p \leqq 2 N} \frac{4}{p^{2}} \cdot \sum_{k}\left|\hat{u}_{p-k}(t)\right|^{2}\right) \cdot \int_{t} \sum_{k>m}^{N} k^{2}\left|\hat{u}_{k}(t)\right|^{2} \\
\quad \leqq 2 \cdot \sum_{N<p \leqq 2 N} \frac{4}{p^{2}} \cdot \max _{t}\left\|u_{N}(\cdot, t)\right\|_{L^{2}(x)}^{2} \cdot\left\|\left(I-P_{m}\right) \frac{\partial u_{N}}{\partial x}\right\|_{L_{\text {loc }}^{2}(x, t)}^{2} \\
\quad \leqq \frac{8}{N} \cdot \text { Const }_{1} \cdot \frac{\text { Const }_{2}}{\varepsilon} .
\end{aligned}
$$

The second integral on the right of (3.21) can be treated similarily and Lemma 3.1 follows with Const ${ }_{12}=4 \cdot\left(\text { Const }_{1} \cdot \text { Const }_{2}\right)^{1 / 2}$.

4. Spectral convergence to the entropy solution. In the last sections we have seen that the spectral approximation, $u_{N}(x, t)$, has a strong limit, $L_{\mathrm{loc}}^{2}-s \lim _{N \rightarrow \infty} u_{N}(x, t)=$ $\bar{u}(x, t)$, which is a weak solution of (1.1). In this section we show that this limit is in fact the unique entropy solution of (1.1) satisfying the entropy inequality (1.2).

THEOREM 4.1. Consider the spectral approximation (2.2) with parameters $(\varepsilon, m)$ that satisfy

$$
\varepsilon=\varepsilon(N) \sim \text { Const. } N^{-2 \beta}, \quad m=m(N) \sim \text { Const. } N^{\beta}, \quad 0<\beta<\frac{1}{4} .
$$

Assume that its solution, $u_{N}(x, t)$ remains uniformly bounded (3.17). Then $u_{N}(x, t)$ converges boundedly almost everywhere to the unique entropy solution of the conservation law (1.1).

Proof. Consider the right-hand side of (3.2), which consists of the sum of two terms, $I I I+I V$. We will show that this sum tends weakly to a negative measure and hence convergence to the entropy inequality (1.2) follows.

As in (3.11) we write $I I I=I I I_{1}+I I I_{2}$, where by Lemma 3.4

$$
\left\|I I I_{1}\right\|_{H_{\text {loc }}^{-1}(x, t)} \leqq \text { Const. }\left\|u_{N}\right\|_{L^{\infty}(x, t)} \cdot N^{\beta-1 / 2} \rightarrow 0,
$$

and in view of our slightly strengthened parametrization (5.1) (compared with (3.18)),

$$
\left\|I I I_{2}\right\|_{L_{\text {loc }}^{1}(x, t)} \leqq \text { Const. } N^{2 \beta-1 / 2} \rightarrow 0, \quad 0<\beta<\frac{1}{4} .
$$


Consequently, the first term on the right of (3.2), III, tends weakly to zero, and we turn to deal with the second one, which is given in (3.14) as $I V=I V_{1}+I V_{2}$. By Lemma 3.5 we have

$$
\left\|I V_{1}\right\|_{H_{\text {loc }}^{-1}(x, t)} \leqq \text { Const. }\left\|u_{N}\right\|_{L^{\infty}(x, t)} \cdot N^{-\beta} \rightarrow 0,
$$

and hence the term $I V_{1}$ also tends weakly to zero. Finally we are left with the term $I V_{2}=-\varepsilon\left(\partial u_{N} / \partial x\right)\left(I-P_{m}\right)\left(\partial u_{N} / \partial x\right)$, which we write as

$$
I V_{2}=-\varepsilon\left(\frac{\partial u_{N}}{\partial x}\right)^{2}+\varepsilon \frac{\partial}{\partial x}\left[u_{N} P_{m} \frac{\partial u_{N}}{\partial x}\right]-\varepsilon u_{N} P_{m} \frac{\partial^{2} u_{N}}{\partial x^{2}} \equiv I V_{21}+I V_{22}+I V_{23}
$$

It follows from (3.20a), that $-\varepsilon\left(\partial u_{N} / \partial x\right)^{2}$ tends weakly to a negative measure,

$$
w \lim _{N \rightarrow \infty}\left[I V_{21} \equiv\left(\frac{\partial u_{N}}{\partial x}\right)^{2}\right] \leqq 0 .
$$

Also, the pessimistic bound

$$
\left\|-\varepsilon \frac{\partial}{\partial x}\left[u_{N} P_{m} \frac{\partial u_{N}}{\partial x}\right]\right\|_{H_{\mathrm{loc}}^{-1}(x, t)} \leqq \varepsilon \cdot\left\|u_{N}\right\|_{L^{\infty}(x, t)} \cdot\left\|P_{m} \frac{\partial u_{N}}{\partial x}\right\|_{L_{\mathrm{loc}}^{2}(x, t)},
$$

yields by Lemma 3.3,

$$
\left\|I V_{22} \equiv \varepsilon \frac{\partial}{\partial x}\left[u_{N} P_{m} \frac{\partial u_{N}}{\partial x}\right]\right\|_{H_{\mathrm{loc}}^{-1}(x, t)} \leqq \text { Const. }\left\|u_{N}\right\|_{L^{\infty}(x, t)} \cdot N^{-\beta} \rightarrow 0,
$$

and hence weak convergence to zero. We conclude with

$$
I V_{23} \equiv-\varepsilon u_{N} P_{m} \frac{\partial^{2} u_{N}}{\partial x^{2}}=-\varepsilon \bar{u} P_{m} \frac{\partial^{2} u_{N}}{\partial x^{2}}-\varepsilon\left(u_{N}-\bar{u}\right) P_{m} \frac{\partial^{2} u_{N}}{\partial x^{2}} \equiv I V_{231}+I V_{232}
$$

Here we have

$$
I V_{231} \equiv-\varepsilon \bar{u} P_{m} \frac{\partial^{2} u_{N}}{\partial x^{2}} \rightarrow 0 \quad \text { (weakly) }
$$

and by Lemma 3.3,

$$
\begin{aligned}
\| I V_{232} & =-\varepsilon\left(u_{N}-\bar{u}\right) P_{m} \frac{\partial^{2} u_{N}}{\partial x^{2}}\left\|_{L_{\mathrm{loc}}^{1}(x, t)} \leqq \varepsilon \cdot\right\| u_{N}-\bar{u}\left\|_{L_{\mathrm{loc}}^{2}(x, t)} \cdot\right\| P_{m} \frac{\partial^{2} u_{N}}{\partial x^{2}} \|_{L_{\mathrm{loc}}^{2}(x, t)} \\
& \leqq \text { Const. } N^{-2 \beta} \cdot N^{2 \beta} \cdot\left\|u_{N}-\bar{u}\right\|_{L_{\mathrm{loc}}^{2}(x, t)} \rightarrow 0 .
\end{aligned}
$$

In summary, we have by (4.2)-(4.10) that the right-hand side tends in the sense of distributions to a negative measure, while the (weak) limit on the left gives us

$$
\frac{\partial}{\partial t}\left(\frac{1}{2} \bar{u}^{2}\right)+\frac{\partial}{\partial x}\left(\frac{1}{3} \bar{u}^{3}\right) \leqq 0
$$

as asserted.

5. Systems of conservation laws. It is instructive to make a comparison between the spectral methods before and after spectral vanishing viscosity was added. Before viscosity was added we had, in (1.6), a coupled system of ODE's in the Fourier space, which amounts to

$$
\frac{d}{d t} \hat{u}_{k}(t)+\frac{1}{2} i k \sum_{p+q=k} \hat{u}_{p}(t) \hat{u}_{q}(t)=0, \quad|k| \leqq N .
$$


In this case the total quadratic entropy was conserved, (1.9), which is responsible to the divergence of the method. After viscosity was added in (2.2), the resulting system in the Fourier space reads

$$
\begin{gathered}
\frac{d}{d t} \hat{u}_{k}(t)+\frac{1}{2} i k \sum_{p+q=k} \hat{u}_{p}(t) \hat{u}_{q}(t)=0, \quad|k| \leqq m, \\
\frac{d}{d t} \hat{u}_{k}(t)+\frac{1}{2} i k \sum_{p+q=k} \hat{u}_{p}(t) \hat{u}_{q}(t)=-\varepsilon k^{2} \hat{u}_{k}(t), \quad|k|>m .
\end{gathered}
$$

An increasing portion of the spectrum is treated here as in the diverging case (5.1). Yet, the added viscosity for the high Fourier modes in (5.2b) is responsible for the correct rate of entropy dissipation (3.6), which in turn implies convergence in the scalar case. In this section we show how to enforce similar entropy dissipation by spectral vanishing viscosity in systems of conservation laws. To this end we proceed as follows.

Consider the conservative system

$$
\frac{\partial}{\partial t} u(x, t)+\frac{\partial}{\partial x}[f(u(x, t))]=0,
$$

which is assumed to be equipped with an entropy function $U(u)$, i.e., a convex function whose Hessian $U_{u u}$ symmetrizes the Jacobian matrix $f_{u}$, e.g., [2], [11]. Using the entropy variables

$$
v \equiv v(u)=\frac{\partial U}{\partial u}(u),
$$

the conservative system (5.3) takes the equivalent symmetric form (see [4], [8], [12])

$$
\frac{\partial}{\partial t} u(x, t)+\frac{\partial}{\partial x}[g(v(x, t))]=0, \quad g(v) \equiv f(u(v)) .
$$

The Fourier approximation of (5.3) will be based on this formulation: together with additional vanishing spectral viscosity we arrive at

$$
\frac{\partial}{\partial t} u_{N}(x, t)+\frac{\partial}{\partial x}\left[P_{N} g\left(v_{N}(x, t)\right)\right]=\varepsilon \frac{\partial}{\partial x}\left[\left(I-P_{m}\right) \frac{\partial}{\partial x} v_{N}(x, t)\right]
$$

where $v_{N}(x, t)$ is the projected vector of entropy variables

$$
v_{N}(x, t)=P_{N}\left[v\left(u_{N}(x, t)\right)\right] .
$$

Multiply $(5.5 \mathrm{a})$ by $v_{N}(x, t)$ and integrate over the $2 \pi$-period: taking into account orthogonality we have

$$
\begin{gathered}
\frac{d}{d t} \int_{-\pi}^{\pi} U\left(u_{N}(x, t)\right) d x+\int_{-\pi}^{\pi} v_{N}(x, t) \frac{\partial}{\partial x}\left[g\left(v_{N}(x, t)\right)\right] d x \\
=\varepsilon \int_{-\pi}^{\pi} v_{N}(x, t) \frac{\partial}{\partial x}\left[\left(I-P_{m}\right) \frac{\partial}{\partial x} v_{N}(\cdot, t)\right] d x .
\end{gathered}
$$

The second integrand on the left is a perfect derivative of the associated entropy flux, and hence its integral vanishes. Integration by parts on the right yields

$$
\frac{d}{d t} \int_{-\pi}^{\pi} U\left(u_{N}(x, t)\right) d x+\varepsilon\left\|\left(I-P_{m}\right) \frac{\partial}{\partial x} v_{N}(\cdot, t)\right\|_{L^{2}(x)}^{2}=0,
$$


which shows that entropy dissipates at the correct rate. In particular, arguing along the lines of Lemma 3.3 and and using the strict convexity of $U(u)$, we conclude that

$$
\sqrt{\varepsilon}\left\|\frac{\partial u_{N}}{\partial x}\right\|_{L_{\mathrm{loc}}^{2}(x, t)} \leqq \text { Const., } \quad \varepsilon m^{2} \sim \text { Const. }
$$

This is analogous to the behavior of the viscous regularization for (5.3) (compare (3.20) in the scalar case).

6. Numerical experiments. The Fourier method with spectral vanishing viscosity was applied to the periodic Burgers' equation (1.1) with $u(x, t=0)=\sin x$ as initial data. The resulting ODE system for the Fourier coefficients (see (5.2))

$$
\begin{gathered}
\frac{d}{d t} \hat{u}_{k}(t)+\frac{1}{2} i k \sum_{p+q=k} \hat{u}_{p}(t) \hat{u}_{q}(t)=-\varepsilon k^{2} \hat{Q}(k) \hat{u}_{k}(t), \quad|k| \leqq N, \\
\hat{Q}(k)= \begin{cases}0, & |k| \leqq m, \\
1, & |k|>m,\end{cases}
\end{gathered}
$$

was integrated up to time $t=1.5$, using the fourth-order Runge-Kutta method.

The number of significant modes was chosen as a fraction of the total number, $m=\theta N$. The numerical experiments indicate, as expected, that the quality of the results is more sensitive to the dependence of $\varepsilon$ on $m$; further investigation is necessary in order to settle this point. In the following examples we have, $\varepsilon m \sim 0.25$, based on considerations of minimizing the total-variation of the numerical solution. With this choice of parameters, Figure 6.1 shows that the numerical solution converges strongly (but not uniformly) to the entropy solution of (1.1). This is in sharp contrast to the oscillatory behavior of the viscosity-free Fourier method in Fig. 6.2, where $\hat{Q}(k) \equiv 0 .{ }^{1}$ Other parameterizations of $\varepsilon$, quoted in Figs. 6.3 and 6.4, demonstrate the sensitivity of the computed solution mentioned earlier.

To improve the quality of these results, the proposed method (6.1a) was implemented with a spectral vanishing viscosity $\hat{Q}(k)$ that is smoothly varying between zero and one, say, for $m / 3 \leqq|k|<m$. Figures 6.5 and 6.6 show that this kind of viscosity prevents the propagation of the Gibbs phenomenon into the whole computational domain that was noticed earlier. This is analogous to the spectral recovery in shadowed regions between propagating linear discontinuities described in [7].

Appendix A. Failure of convergence with post-processing. In practical applications the classical Fourier method is often coupled with certain smoothing procedures the purpose of which is to gain spectral convergence that otherwise might be lost. In a typical case, the solution is post-processed via a convolution with a smoothing kernel $Q_{N} \equiv Q_{N}(x)$

$$
Q_{N} * u_{N}(x, t)=\sum_{k=-N}^{N} \hat{q}_{k} \hat{u}_{k}(t) e^{i k x}, \quad 0 \leqq \hat{q}_{k} \leqq \hat{q}_{0}=1, \quad \hat{q}_{k} \equiv \hat{q}_{k, N} .
$$

In order to maintain spectral accuracy, the convolution with such smoothing kernel should be highly accurate with that of Dirac's $\delta$-distribution. We shall make a minimal assumption in this direction, requiring that for all functions $\varphi(x)$ in the Wiener class, $\sum_{k=-\infty}^{\infty}|\hat{\varphi}(k)|<\infty$, we have

$$
\left\|\left(\delta-Q_{N}\right) * \varphi(x)\right\|_{L^{\infty}(x)} \leqq \sum_{k=-N}^{N}\left|1-\hat{q}_{k, N}\right| \cdot|\hat{\varphi}(k)| \underset{N \rightarrow \infty}{\longrightarrow} 0 .
$$

\footnotetext{
${ }^{1}$ In fact, a slight amount of dissipation was introduced in this case due to the time integrator.
} 
40

E. TADMOR
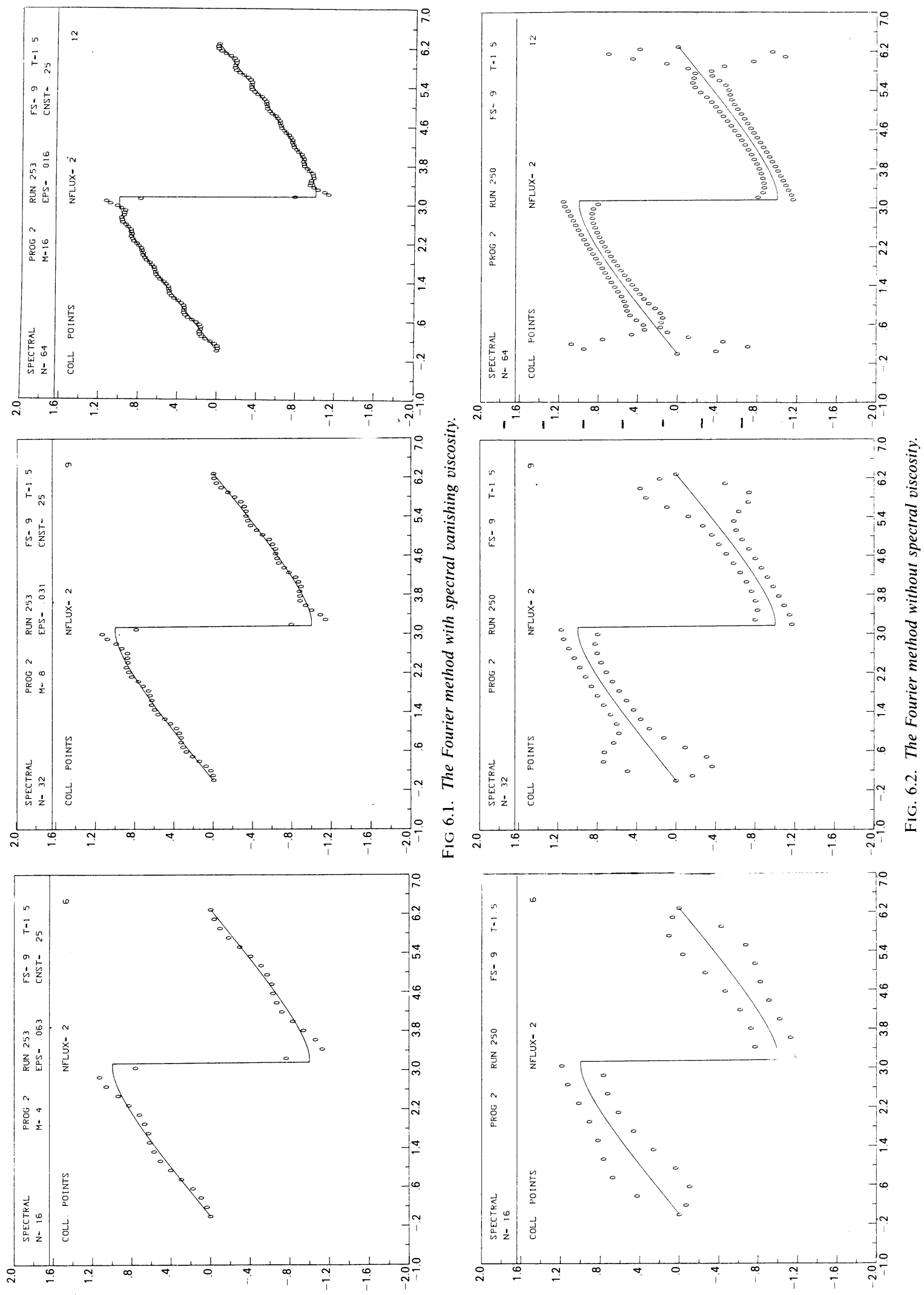

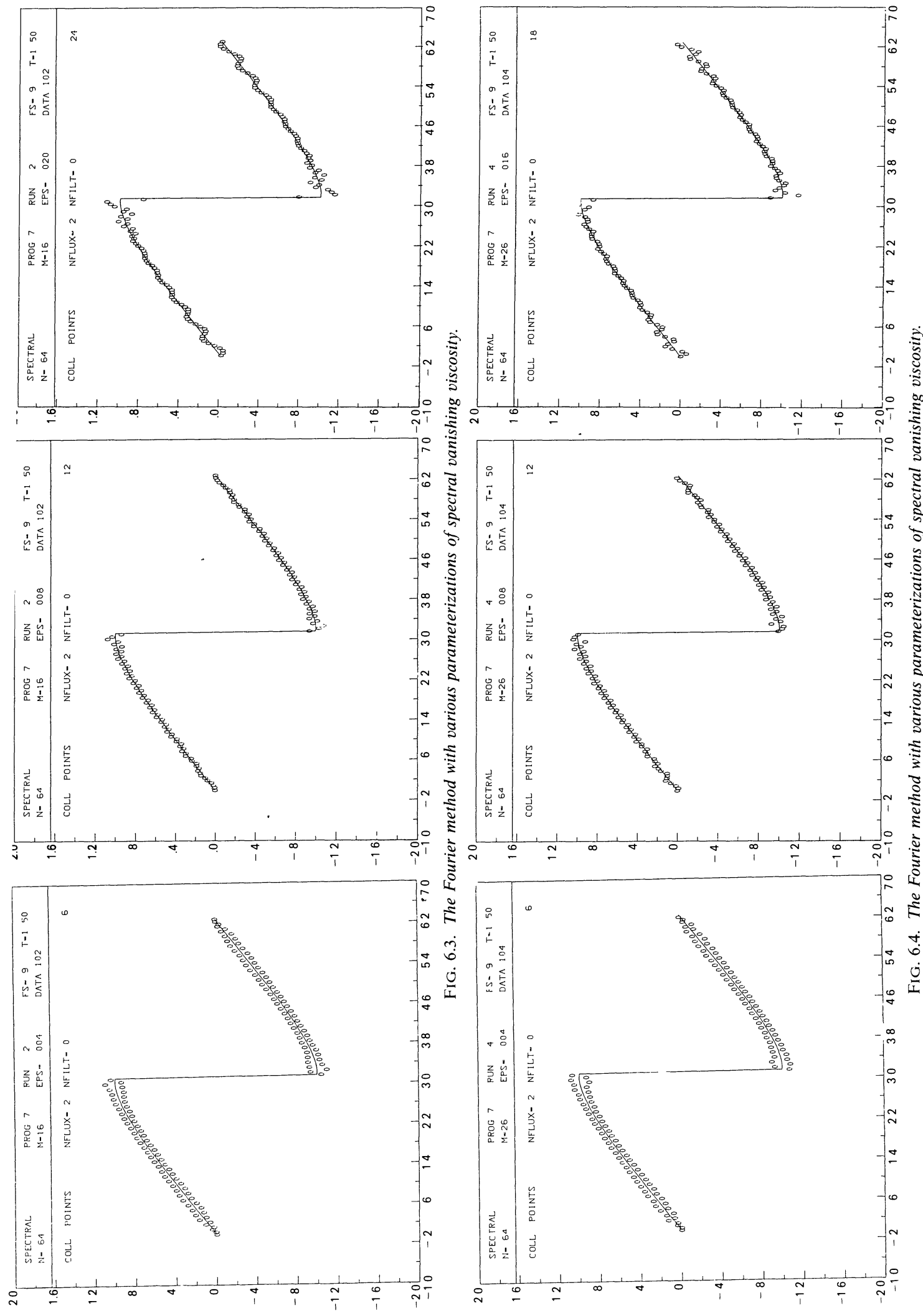


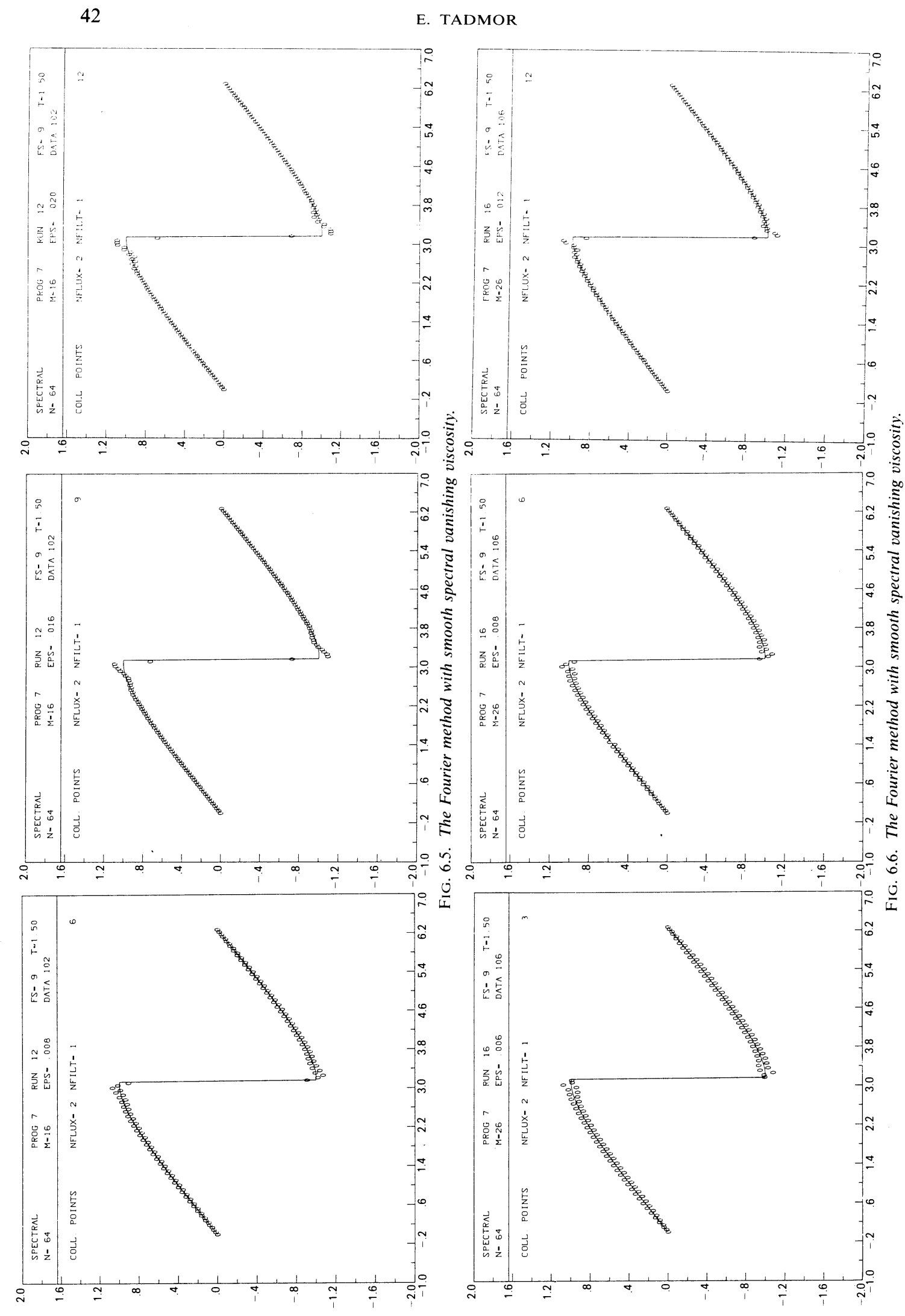


Such smoothing procedure enables, for example, spectral recovery of solutions to linear hyperbolic problems in the presence of propagating initial discontinuities (e.g., [1], [7]).

We will show that the smoothed version of the Fourier approximation to the nonlinear inviscid Burgers' equation (1.1),

$$
\frac{\partial}{\partial t} u_{N}(x, t)+\frac{\partial}{\partial x}\left[\frac{1}{2} P_{N}\left(Q_{N} * u_{N}(x, t)\right)^{2}\right]=0,
$$

where the solution (rather than the flux) is convolved with kernels satisfying (A.2), does not converge to the entropy solution of (1.1).

To this end we convolve (A.3) with $Q_{N}$ to find that $w_{N}(x, t)=Q_{N} * u_{N}(x, t)$ satisfies

$$
\frac{\partial}{\partial t} w_{N}(x, t)+Q_{N} * \frac{\partial}{\partial x}\left[\frac{1}{2} P_{N} w_{N}^{2}(x, t)\right]=0 .
$$

Multiplying (A.4) by $u_{N}(x, t)$ and integrating over the $2 \pi$-period, we obtain

$$
\int_{x} u_{N} \frac{\partial w_{N}}{\partial t} d x=-\int_{x} Q_{N} * u_{N} \frac{\partial}{\partial x}\left[\frac{1}{2} P_{N} w_{N}^{2}\right] d x
$$

or, in view of orthogonality,

$$
\frac{1}{2} \frac{d}{d t} \int_{x} u_{N} Q_{N} * u_{N} d x=-\int_{x} w_{N} \frac{\partial}{\partial x}\left[\frac{1}{2} w_{N}^{2}\right] d x=0 .
$$

Thus with $Q_{N}^{1 / 2} * \varphi \equiv \sum_{k=-N}^{N} \hat{q}_{k}^{1 / 2} \cdot \hat{\varphi}(k)$ we have

$$
\left\|Q_{N}^{1 / 2} * u_{N}(\cdot, t)\right\|_{L^{2}(x)}^{2}=\sum_{k=-N}^{N} \hat{q}_{k}\left|\hat{u}_{k}(t)\right|^{2} \equiv \text { Const. }
$$

This implies that our smoothed approximation $w_{N}(x, t)=Q_{N} * u_{N}(x, t)$ converges to a weak limit $w \lim _{N \rightarrow \infty} w_{N}(x, t)=\bar{w}(x, t)$. Now, suppose that $\bar{w}(x, t)$ is a weak solution of $(1.1)$; this will lead us to the conservation of $\int_{x} \bar{w}^{2}(x, t) d x$, which shows that $\bar{w}(x, t)$ is not the entropy solution (1.1). Indeed, if $\bar{w}(x, t)$ satisfies (1.1), then by (A.4), $Q_{N} * P_{N} w_{N}^{2}(x, t) \equiv Q_{N} * w_{N}^{2}(x, t)$ tends weakly to $\bar{w}^{2}(x, t)$

$$
w \lim _{N \rightarrow \infty} Q_{N} * P_{N} w_{N}^{2}(x, t)=\bar{w}^{2}(x, t) .
$$

It follows from (A.5) that $\left\|w_{N}^{2}(\cdot, t)\right\|_{L^{1}(x)}$ is bounded, and together with (A.2) this implies that for all $C_{0}^{\infty}$-test functions $\varphi(x)$ we have

$$
\int_{x} \varphi(x)\left(\delta-Q_{N}\right) * w_{N}^{2}(x, t) d x \leqq \text { Const. }\left\|\left(\delta-Q_{N}\right) * \varphi(x)\right\|_{L^{\infty}(x)} \underset{N \rightarrow \infty}{\longrightarrow} 0 .
$$

Adding this to (A.6), we conclude that $P_{N} w_{N}^{2}(x, t)$, and hence $w_{N}^{2}(x, t)$ tends weakly to $\bar{w}^{2}(x, t)$. Consequently $\bar{w}(x, t)$ is the $L^{2}$-strong limit of our smoothed approximation

$$
s \lim _{N \rightarrow \infty} Q_{N} * u_{N}(x, t)=\bar{w}(x, t) .
$$

Finally, in view of (A.5) we can apply (A.2) to find that

$$
\left\|Q_{N} * u_{N}(x, t)-Q_{N}^{1 / 2} * u_{N}(x, t)\right\|_{L^{2}(x)}^{2}=\sum_{k=-N}^{N}\left|1-\hat{q}_{k, N}^{1 / 2}\right|^{2} \cdot \hat{q}_{k, N} \cdot\left|\hat{u}_{k}(t)\right|^{2} \underset{N \rightarrow \infty}{\longrightarrow} 0 .
$$

From (A.8) and (A.9) it follows that $\bar{w}(x, t)$ is the strong limit of $Q_{N}^{1 / 2} * u_{N}(x, t)$, and the strong limit of (A.5) tells us that $\int_{x} \bar{w}^{2}(x, t) d x$ is conserved in time, which completes our asserted contradiction. We summarize by stating the following theorem. 
THEOREM A1. The Fourier method (A.3) which employs any smoothing kernel satisfying (A.2) does not converge to the entropy solution of (1.1), (1.2).

\section{REFERENCES}

[1] S. Abarbanel, D. Gottlieb, and E. Tadmor, Spectral methods for discontinuous problems, in Numerical Methods for Fluid Dynamics II, K. W. Morton and M. J. Baines, eds., Oxford University Press, London, 1986, pp. 129-153.

[2] K. O. Friedrichs AND P. D. LAx, Systems of conservation laws with a convex extension, Proc. Nat. Acad. Sci. U.S.A., 68 (1971), pp. 1686-1688.

[3] D. Gottlieb and S. Orszag, Numerical Analysis of Spectral Methods: Theory and Applications, CBMS-NSF Regional Conference Series in Applied Mathematics 26, Society for Industrial and Applied Mathematics, Philadelphia, PA, 1977.

[4] A. HARTEN AND P. D. LAX, A random choice finite difference scheme for hyperbolic conservation laws, SIAM J. Numer. Anal., 18 (1981), pp. 289-315.

[5] H. O. Kreiss And H. Oliger, Stability of the Fourier method, SiAM J. Numer. Anal., 16 (1979), pp. 421-433.

[6] P. D. LAx, Hyperbolic Systems of Conservation Laws and the Mathematical Theory of Shock Waves, ABMS-NSF Regional Conference Series in Applied Mathematics 11, Society for Industrial and Applied Mathematics, Philadelphia, PA, 1972.

[7] A. Majda, J. McDonough, And S. Osher, The Fourier method for nonsmooth initial data, Math. Comp., 32 (1978), pp. 1041-1081.

[8] M. S. Mock, Systems of conservation laws of mixed type, J. Differential Equations, 37 (1980), pp. $70-88$.

[9] J. VON NEUMANN AND R. D. RiCHTMYER, A method for the numerical calculations of hydrodynamical shocks, J. Appl. Phys., 21 (1950), pp. 232-237.

[10] E. TADMOR, The exponential accuracy of Fourier and Chebyshev differencing methods, SIAM J. Numer. Anal., 23 (1986), pp. 1-10.

[11] - Entropy functions for symmetric systems of conservation laws, Math. Anal. Appl., 122 (1987), pp. 355-359.

[12] - The numerical viscosity of entropy stable schemes for systems of conservation laws, J. Math. Comp., 48 (1987), pp. 91-103.

[13] - Stability analysis of finite difference, pseudospectral, and Fourier-Galerkin approximations for time-dependent problems, SIAM Rev., 29 (1987), pp. 525-556.

[14] L. TARTAR, Compensated compactness and applications to partial differential equations, in Research Notes in Mathematics 39, Nonlinear Analysis and Mechanics, Heriot-Watt Symposium, Vol. 4, R. J. Knopps, ed., Pitman Press, Boston, London, 1975, pp. 136-211.

[15] - Introduction à la méthode de compacité par compensation, in Nonlinear Hyperbolic Problems, C. Carasso, P.-A. Raviart and D. Serre eds., Proceedings of an advanced research workshop on Hyperbolic Problems: Theory, Numerical Methods and Applications, Saint-Etienne, 1986, Lecture Notes in Mathematics, Springer-Verlag, Berlin, New York, 1987. 